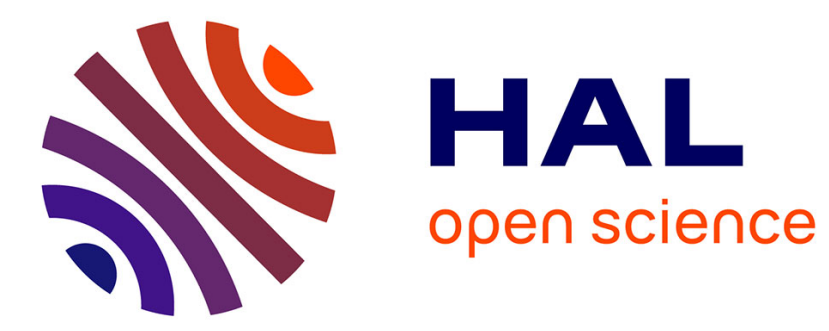

\title{
Lagrangian scheme for scalar advection-diffusion - Application to pollutant transport
}

\author{
Benoît Trouette, Georges Halim Atallah, Stéphane Vincent
}

\section{To cite this version:}

Benoît Trouette, Georges Halim Atallah, Stéphane Vincent. Lagrangian scheme for scalar advectiondiffusion - Application to pollutant transport. Turbulence and Interactions, 2021. hal-03129042

\section{HAL Id: hal-03129042 \\ https://hal.science/hal-03129042}

Submitted on 2 Feb 2021

HAL is a multi-disciplinary open access archive for the deposit and dissemination of scientific research documents, whether they are published or not. The documents may come from teaching and research institutions in France or abroad, or from public or private research centers.
L'archive ouverte pluridisciplinaire HAL, est destinée au dépôt et à la diffusion de documents scientifiques de niveau recherche, publiés ou non, émanant des établissements d'enseignement et de recherche français ou étrangers, des laboratoires publics ou privés. 


\title{
Lagrangian scheme for scalar advection-diffusion - Application to pollutant transport
}

\author{
Benoît Trouette ${ }^{1}$, Georges Halim Atallah ${ }^{1,2}$, and Stéphane Vincent ${ }^{1}$ \\ 1 Université Paris-Est, Laboratoire Modélisation et Simulation Multi Echelle, \\ MSME UMR 8208, CNRS, UPEC, UPEM, F-77454, Marne-la-Vallée, France \\ 2 INRS, French National Research and Safety Institute for the Prevention of Occupational \\ Accidents and Diseases, Lab. Aeraulic engineering, Vandœuvre, France \\ \{benoit.trouette; georges.halimatallah; stephane.vincent\}@u-pem.fr
}

\begin{abstract}
A Lagrangian scheme devoted to the approximation of advection term in advection-diffusion equation (ADE) is proposed to deal with large values of Péclet number. Advection and diffusion of circular concentration in a vortex flow are considered for validation purpose. The Lagrangian scheme reduces the numerical diffusion to almost computer error and provides better results than other Eulerian classical schemes of the literature. The injection of a pollutant in a cavity is finally illustrated.
\end{abstract}

Keywords: Lagrangian scheme · advection-diffusion · large Pe number

\section{Introduction}

The tracking of pollutants in gas and liquid is a major problem to address in atmospheric environment, air quality characterization and industrial material processes. The design of schemes for the hyperbolic advection term of the transport equation for pollutant concentration has been widely studied, with mostly Eulerian schemes relying on splines reconstruction, high-order spectral, finite difference and finite volume schemes, combined with limiters belonging to the class of TVD or WENO approaches. Reviews and comparisons of these schemes are for example given in [5] [6]. The conclusion of all studies if that when the molecular or turbulent diffusion is low compared to the advection, all schemes are diffusive or dispersive, providing unexpected spreading or unphysical oscillations of the numerical solutions. In the present work, a new Lagrangian scheme [6] is proposed for the advection-diffusion equations that avoids diffusion and respects the monotonicity of the solution.

\section{Model and numerical methods}

The framework of the present work is the simulation of incompressible fluid flows with a Finite-Volume method on Cartesian staggered grids. The advection-diffusion equation (ADE) of a quantity $\Phi$ (temperature, pollutant concentration, ...) is considered. $\Gamma$ is the diffusivity and $\vec{u}$ the fluid velocity, either prescribed or given by the last iteration of the Navier-Stokes resolution. Classical Eulerian Centered, Upwind, 
Quick, TVD or WENO schemes are generally used for discretizing the advection term. Solving the ADE with centered schemes introduce oscillations and non bounded solutions for a low diffusivity coefficient $\Gamma$ or high Péclet cell number values, given by $P e=\|\vec{u}\| \Delta x / \Gamma$. On the contrary, low order upwind schemes induce important numerical diffusion that alter the physical meaning of the solution, as centered schemes do. A Sequential Operator Splitting (SOS) method $[2,4]$ is utilized. Introducing a time step $\Delta t, \mathrm{ADE}$ is split in time into two substeps: advection

$$
\frac{\partial \Phi}{\partial t}+\vec{\nabla} \cdot(\vec{u} \Phi)=0
$$

is first solved along the time interval $] t, t+\Delta t]$, providing intermediate solution $\Phi^{\star}$. Then, diffusion

$$
\frac{\partial \Phi}{\partial t}-\vec{\nabla} \cdot(\Gamma \vec{\nabla} \Phi)=0
$$

is solved with initial condition $\Phi(t)=\Phi^{\star}(t+\Delta t)$. It provides the approximated solution of $\Phi(t+\Delta t)$. The SOS is second-order accurate at each time step [2]. More sophisticated SOS can be found in [2,4]. Let us consider $\Phi^{n}=\Phi\left(t^{n}\right)$ as the discrete value of $\Phi$ at time $t^{n}=t^{0}+n \Delta t, n$ being the iteration number and $\Delta t=t^{n+1}-t^{n}$ the time step. In practice, the intermediate solution $\Phi^{\star}$ after advection is updated solving Eq. (1) with an explicit Lagrangian scheme (see below) while Eq. (2) is discretized with an implicit Eulerian centered scheme and direct MUMPS [1] or preconditioned MILU-BICGSTAB II iterative solvers are used to obtain solutions. In addition to the Lagrangian scheme developed in this paper, a non-conservative Weno scheme of order 5 [3] will be used, coupled with a third order Runge-Kutta time integration. For comparison, results obtained with a QUICK scheme are also presented. The Lagrangian scheme for ADE is an extension of VSM scheme from [6]. A number of $M$ markers (Lagrangian particles), located at $\vec{X}_{m}$ and bringing volumes $\delta V_{m}$, are seeded in the Eulerian grid (Fig. 1) devoted to solving of conservation equations. Initially, markers are equality placed in each cell according to a number of particles per direction and per cell $(p p d p c)$. A simulation then handles $p p d p c^{d} \times N_{x} \times N_{y} \times N_{z}^{d-2}$ Lagrangian objects, where $d$ is the space dimension and $N_{x}, N_{y}$ and $N_{z}$ are the numbers of scalar cells in each space direction. Furthermore, the markers carry the local information, $\phi_{m}$, of the Eulerian field $\Phi$. At initial time $\phi_{m}^{0}=\Phi^{0}\left(\vec{X}_{m}^{0}\right)$. The markers are then advected solving the Lagrangian equation $\frac{d \vec{X}_{m}}{d t}=\left.\vec{u}\right|_{\vec{X}_{m}}$. The material derivative on marker position is integrated in time with a second order Runge-Kutta scheme. The velocities at particle positions follow a linear $Q 1$ interpolation. As illustrated in Fig. 1 for marker 2 , the volume $\delta V_{m}$ of a marker can contribute to the computation of $\Phi^{\star}$ on neighboring cells. The value of $\Phi^{n+1}$ is then obtained solving the unsteady diffusion equation (2). Finally, the local (marker) information is updated according to the variation of $\Phi$ during the diffusion step at the particle position:

$$
\left.\frac{\partial \phi_{m}}{\partial t}\right|_{\vec{X}_{m}^{n+1}}=\left.\frac{\partial \Phi}{\partial t}\right|_{\vec{X}_{m}^{n+1}}
$$

The number of particles per cell, $M_{c}$, varies over time. It can increase or decrease according to local streamline convergence or divergence. It can be interesting to remove or add particles in each cell in order to save computational time and maintain 


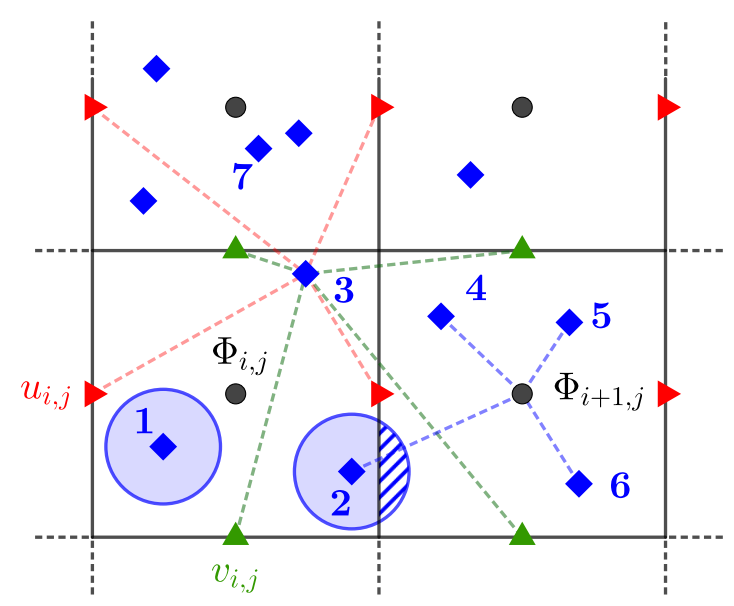

Fig. 1. Sketch of the Lagrangian particles (blue diamond) on a 2D staggered grid. Velocities at particles position are interpolated with surrounding grid velocities (see marker 3 for example, red left triangle and green triangle stand for horizontal and vertical components, respectively). The Eulerian information is computed with averages of markers included in the corresponding cell (see cell $\Omega_{i+1, j}$ for example).

physical relevancy of the solution. This way, $M_{c}$ is reduced to a constant value at each iteration by using the following treatment:

- In each cell where $M_{c}>p p d p c^{d}$, the distances between particles and its neighbors are computed for every pair. The particle having the lower distance (according to a norm, $L_{2}$ for example) is removed (particle 7 in Fig. 1) and this procedure is applied until $M_{c}=p p d p c^{d}$.

- In cells where $M_{c}<p p d p c^{d}$, new particles are randomly introduced until $M_{c}=$ $p p d p c^{d}$. By noting $M_{c}^{0}$ the initial number of markers, we have to insert $M_{i}=$ $p p d p c^{d}-M_{c}^{0}$ particles.

As discussed in the next section, these reseeding operations can lead to spurious effect on isovalues of the scalar field.

\section{Advection-diffusion of a concentration peak}

A peak of concentration is placed in a domain defined by $(x, y) \in[0, L]^{2}$ with velocity field $\vec{u}(x, y)=-a\left(y-y_{c}\right) \vec{e}_{x}+a\left(x-x_{c}\right) \vec{e}_{y}$ corresponding to a block rotation motion around the fixed point $\left(x_{c}, y_{c}\right)$. With $a=\pi / 2$ and $x_{c}=y_{c}=L / 2$, a marker placed in this field needs $T=4$ seconds to make a complete turn and return back to its initial position. In this velocity field, a peak of concentration of radius $R$ is initialized such that

$$
\Phi(r, t=0)=\left\{\begin{array}{rr}
\frac{R-r}{R} & \text { if } r<R \\
0 & \text { otherwise }
\end{array}\right.
$$


where $r=\sqrt{\left(x-x_{p}\right)^{2}+\left(y-y_{p}\right)^{2}}$ is the radial coordinate centered around $\left(x_{p}, y_{p}\right)$ the position of the peak center. At the initial time, $\left(x_{p}, y_{p}\right)=(L / 2,3 L / 4)$. The analytical solution over time is given by

$$
\Phi(r, t)=\sum_{n=1}^{\infty} A_{n} J_{0}\left(\lambda_{n} r\right) \exp \left(-\lambda_{n}^{2} \Gamma t\right)
$$

where $J_{0}$ is the zero ${ }^{t} h$ order Bessel function of the first kind and $\lambda_{n}$ the $n^{\text {th }}$ root of $J_{0}(\lambda)=0$. The $A_{n}$ coefficients are obtained as:

$$
\begin{aligned}
A_{n}=\frac{2 R J_{1}\left(R \lambda_{n}\right)}{\lambda_{n} J_{1}\left(\lambda_{n}\right)^{2}} & -\frac{2}{R J_{1}\left(\lambda_{n}\right)^{2} \lambda_{n}^{3}}\left(\left(R \lambda_{n}\right)^{2} J_{1}\left(R \lambda_{n}\right)-\right. \\
& \left.\frac{\pi R \lambda_{n}}{2}\left[H_{0}\left(R \lambda_{n}\right) J_{1}\left(R \lambda_{n}\right)-H_{1}\left(R \lambda_{n}\right) J_{0}\left(R \lambda_{n}\right)\right]\right)
\end{aligned}
$$

with $J_{1}$ the first order Bessel function of the first kind, and $H_{0}$ and $H_{1}$ the Struve functions of order 0 and 1 , respectively. The numerical solutions obtained with different schemes are compared to this reference solution after a simulation time of $T=4$ seconds and the diffusion coefficient $\Gamma$ varies from $10^{-4}$ to $10^{-6} \mathrm{~m}^{2} / \mathrm{s}$. The domain is discretized with $N_{x}=N_{y}=N$ and $\Delta t$ is chosen according to CFL condition defined by $\frac{a}{2} \Delta t / \Delta x(a / 2$ is the velocity at the peak center). A first set of results is given in Fig. 2 for $\Gamma=10^{-6} \mathrm{~m}^{2} / \mathrm{s}$ and different schemes. On one hand, The QUICK scheme
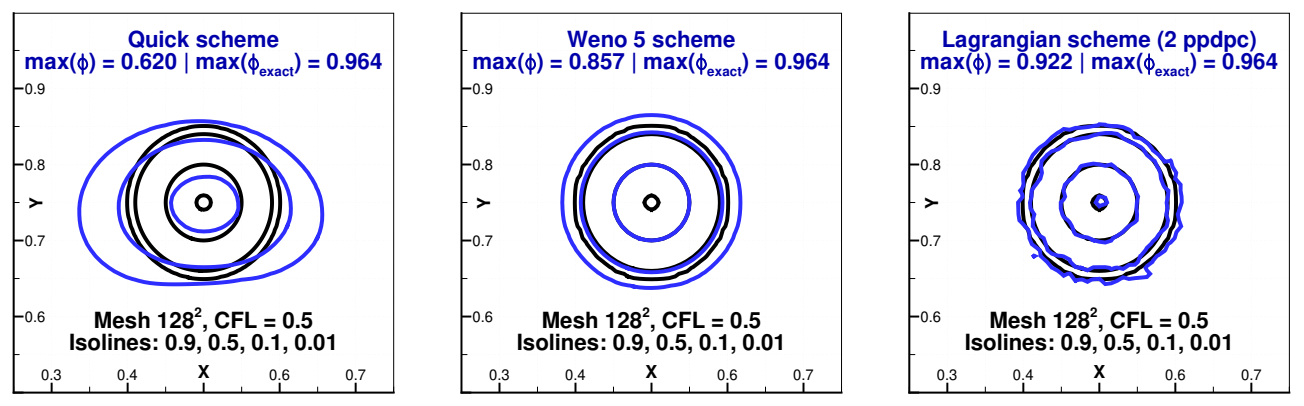

Fig. 2. Zoom on the numerical (blue lines) and reference solutions (black) after one turn for different schemes on a $N^{2}=128^{2}$ mesh and for $\Gamma=10^{-6} \mathrm{~m}^{2} / \mathrm{s}$.

introduces an excessive numerical diffusion and is sensitive to the advection direction. The numerical diffusion is reduced with the Weno5 scheme. On the other hand, oscillations disrupt the numerical solution of the Lagrangian scheme but this latter do not introduce numerical diffusion and presents, on this mesh, a better solution than the other schemes. Note that the Lagrangian scheme, with 2 particles per direction and per cell $(p p d p c)$, is able to represent $95 \%$ of the peak value. The second set of results (Fig. 3) presents, on the same mesh, the results obtained with the Lagrangian scheme and different values of $p p d p c$. The first observation is that the oscillations can be reduced increasing by the $p p d p c$ value. In the same time, the quality of the solution increase, i.e. $99.2 \%$ of the peak value is correctly represented for $p p d p c=8$. It has 

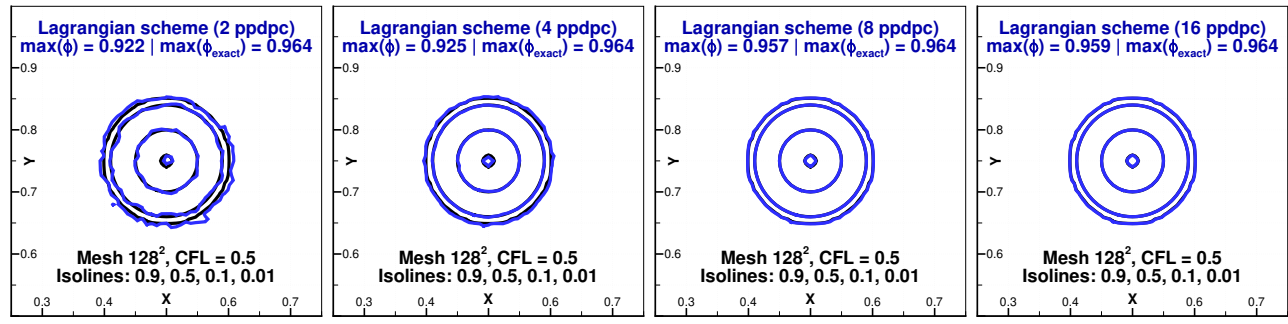

Fig. 3. Zoom on the numerical (blue lines) and reference solutions (black) after one turn for different values of $p p d p c$ on a $N^{2}=128^{2}$ mesh and for $\Gamma=10^{-6} \mathrm{~m}^{2} / \mathrm{s}$.

been verified that the oscillations come from the reseeding procedures. Indeed, all the markers follow circle trajectories and come back to their initial position after time $T$. Removing reseeding procedures in this particular rotation motion case suppress the oscillations even with a small $p p d p c$ value. However, in practical cases, enrichment is mandatory to balance particle lack in sheared zones.

\section{Injection of a pollutant in a cavity}

The Lagrangian scheme is applied to polluted air injected into a rectangular enclosure containing the same fluid at rest. A round jet enters with a flow rate of $40 \ell / \mathrm{min}$. Gas exits through an open circular outlet at atmospheric pressure. The Reynolds number based on the injector diameter is $R e=1500$. The numerical solution is obtained on a mesh with $64 \times 32 \times 32$ cells and without any turbulence modeling. The dynamical solution is used in the ADE equation. The Figure 4 presents the concentration fields is observed after 1.5 second. Iso-concentration for the Weno5 and Lagrangian with 4
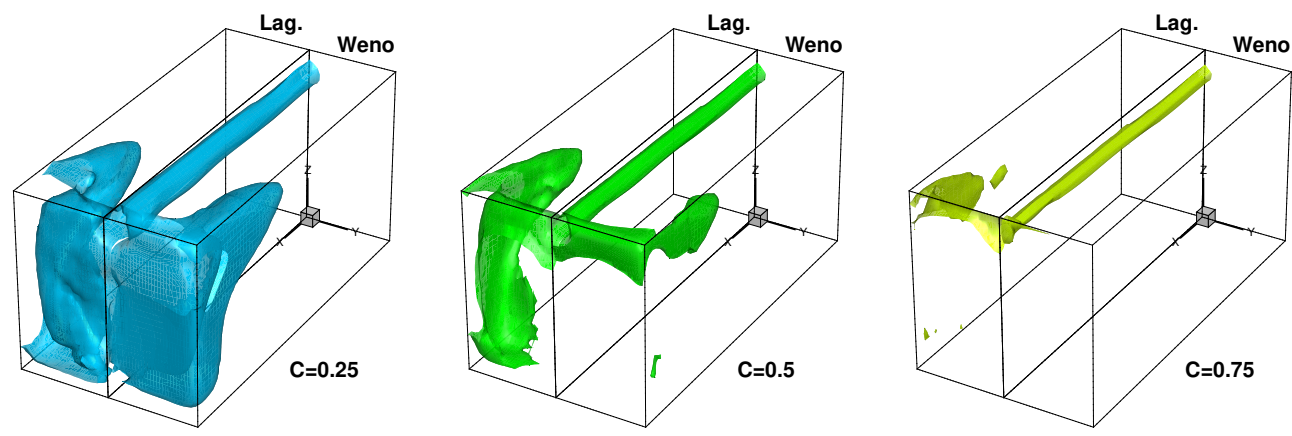

Fig. 4. Iso-concentration $C=0.25,0.5$ and 0.75 plotted for Lagrangian and Weno5 scheme at $1.5 \mathrm{~s}$.

ppdpc schemes are shown. The left part of the cavity presents the iso-concentration obtained with the Lagrangian scheme while the right part provides the Weno5 results. The numerical diffusion introduced by the Weno scheme prevents it form picking up 
the signal correctly while the Lagrangian scheme is able to represent high values of the concentration field.

\section{Concluding remarks}

The efficiency of a fully Lagrangian scheme has been demonstrated for the resolution of an ADE. It is even more effective than the ratio between advection and diffusion is important. The overcost of the particle tracking method used by the Lagrangian scheme is counterbalanced by the fact that the advection process does not introduce numerical diffusion, respect monotonicity of the solution and allows coarser meshes to use in comparisson to classical Eulerian schemes. The major drawback of the Lagrangian approach is the spurious oscillations introduced by the reseeding procedures. This will be considered in future works, as well as local varying Lagrangian seeding of particles in order to reduce the global cost of the scheme.

\section{Acknowledgment}

The authors are grateful for access to the computational facilities of the French GENCI under project number A0032B06115.

\section{References}

1. Amestoy P. R., Duff I. S., Koster J., L'Excellent J.-Y. A Fully Asynchronous Multifrontal Solver Using Distributed Dynamic Scheduling. SIAM Journal on Matrix Analysis and Applications 23, pp 15-41 (2001)

2. Chertock A., Kurganov A., Petrova G. Fast explicit operator splitting method for convection-diffusion equations. International Journal for Numerical Methods in Fluids 59, pp. 309-332 (2006)

3. G.-S. Jiang, S.-W. Shu F. Efficient Implementation of Weighted ENO Schemes. Journal of Computational Physics, 126, pp. 202-228 (1996)

4. Geiser J., Elbiomy M. Splitting Method of Convection-Diffusion Methods with Disen- tanglement methods. Humboldt-Universität zu Berlin, Mathematisch-Naturwissenschaftliche Fakultät II, Institut für Mathematik (2011)

5. Nguyen K., Dabdub D. Two-level time-marching scheme using splines for solving the advection equation. Atmospheric Environment, 35, pp. 1627-1637 (2001)

6. Vincent S., Balmigère G., Caltagirone J.-P., Meillot E. Eulerian-Lagrangian multiscale methods for solving scalar equations - Application to incompressible two-phase flows. Journal of Computational Physics 229, pp 73-106 (2010) 